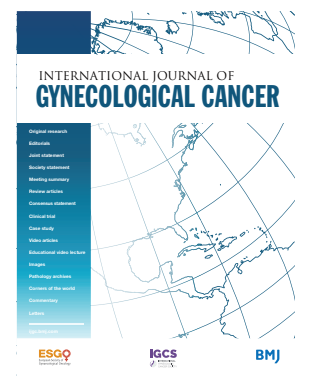

\title{
Recurrence of vulvar squamous cell carcinoma as an undifferentiated sarcomatoid carcinoma
}

\author{
Case Presenter: \\ Chiara Comerio \\ Department of Obstetrics and Gynecology, San Gerardo Hospital, University of Milano-Bicocca, \\ Monza, Italy \\ Pathologist: \\ Marta Jaconi \\ Department of Pathology, San Gerardo Hospital, University of Milano-Bicocca, Monza, Italy \\ Benedetta Zambetti \\ Department of Obstetrics and Gynecology, San Gerardo Hospital, University of Milano-Bicocca, \\ Monza, Italy \\ Mariachiara Paderno
Department of Obstetrics and Gynecology, San Gerardo Hospital, University of Milano-Bicocca,
Monza, Italy \\ Maria Gabriella Valente \\ Department of Pathology, San Gerardo Hospital, University of Milano-Bicocca, Monza, Italy \\ Discussant: \\ Alessandro Buda \\ Department of Obstetrics and Gynecology, San Gerardo Hospital, University of Milano-Bicocca, \\ Monza, Italy
}

\section{Correspondence to} Dr Alessandro Buda, Department of Obstetrics and Gynecology, San Gerardo Hospital, University of MilanoBicocca, 20900 Monza, Italy; alebuda1972@gmail.com

Accepted 20 November 2020

Check for updates

(C) IGCS and ESGO 2021. No commercial re-use. See rights and permissions. Published by BMJ.

To cite: Comerio $\mathrm{C}$, Jaconi $\mathrm{M}$, Zambetti B, et al. Int J Gynecol Cancer 2021;31:627-630.

\section{CASE PRESENTATION}

In November 2019, a 75 year-old woman with a history of diabetes mellitus and major depression with psychotic features underwent a radical vulvectomy, including the resection of the external meatus of the urethra and the distal third of the vagina, for a welldifferentiated infiltrating squamous cell vulvar carcinoma, stage II (Figure 1A,B). In addition, bilateral groin sentinel lymph node biopsy was performed. Resection margins were free of disease, with the closest lateral margin of $4 \mathrm{~mm}$, and the closest deep margin of $2 \mathrm{~mm}$. Bilateral sentinel lymph nodes (two lymph nodes each side) were negative. The post-operative course was uneventful, and the patient was discharged on day four after surgery.

In view of the patient's age, the instability of her psychiatric condition, and associated comorbidities, the multidisciplinary team recommended no further adjuvant therapy. In April 2020, at the follow-up visit the patient reported vulvar itching and discomfort. The vaginal vestibule had evidence of a $4.5 \times 3 \mathrm{~cm}$ exophytic ulcerated mass with an irregular purple to brown surface (Figure 2). A repeat biopsy was not performed as findings were considered consistent with the previous malignancy. A PET/CT was

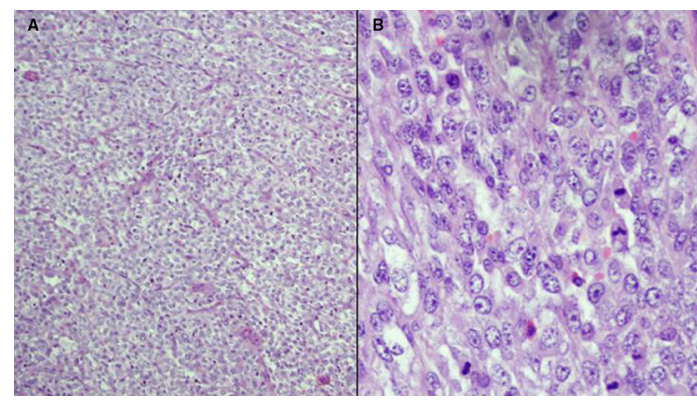

Figure 1 (H\&E, 2x A, 20x B). Microscopic appearance of the original lesion, which had a conventional squamous morphology with foci of keratinization and moderate nuclear atypia, which led to a final diagnosis of moderately differentiated keratinizing squamous cell carcinoma.

performed, and this did not demonstrate any suspicious distant metastases. Therefore, the surgical removal of the local recurrence was recommended. She underwent a radical excision of the vulvo-vaginal lesion with a correction of the local defect with plastic reconstruction which included elevation of a bilateral lotus petal flap. (Figure 3 ) In our experience, this 


\section{Case study}

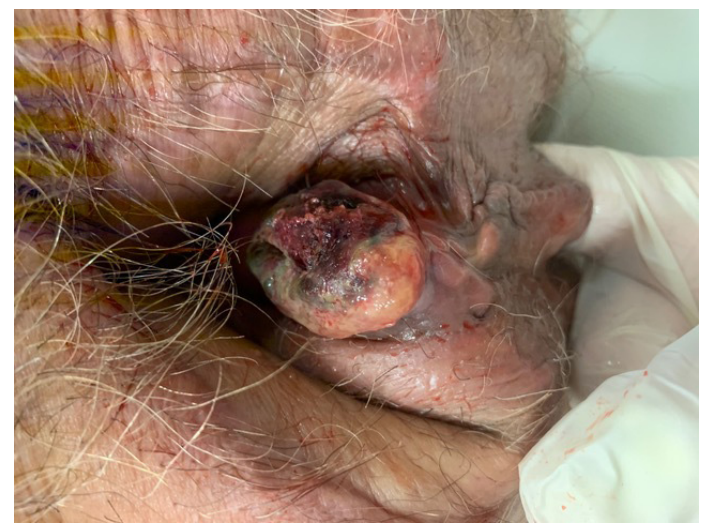

Figure 2 The vulvo-vaginal recurrence.

versatile reconstructive technique provides a low complication rate and represents the treatment of choice for vulvar reconstruction in the absence of a large tissue defect, leading to the best esthetic and functional results. ${ }^{1}$

Histological evaluation of the recurrent lesion revealed that it was composed of medium-sized neoplastic cells growing in solid sheets, with ample areas of hemorrhage and necrosis, characterized by clear cytoplasm and atypical rounded nuclei, with conspicuous nucleoli and a high mitotic index (Ki67 80\%, with many visible mitoses) (Figure 4). Immunohistochemical profiling of the cells showed diffuse reactivity for pancytokeratin and CK7 (Figure 5), while high molecular weight cytokeratin, EMA, and CK19 were negative. Other squamous cell markers were also negative, with an absence of reactivity for p40 and only focal and weak for p63 (Figure 6). A panel for vascular markers revealed strong and diffuse positivity for CD31 (Figure 7), weak and focal positivity for FLI1 and ERG, while no reactivity was shown for CD34 and factor VIII. Vulvar melanoma was ruled out with the observation of negative $S 100$ and SOX10. The neoplasia had a maximum depth of infiltration measuring $18 \mathrm{~mm}$, with $6 \mathrm{~mm}$ of stroma between the mass and the resection margin: the closest lateral margin was toward the vagina, with a distance of $2 \mathrm{~mm}$. No lymphovascular invasion was observed.

After careful consideration of morphology, immunohistochemistry, and clinical setting, the final diagnosis was of undifferentiated carcinoma with sarcomatoid features, to be ascribed to a

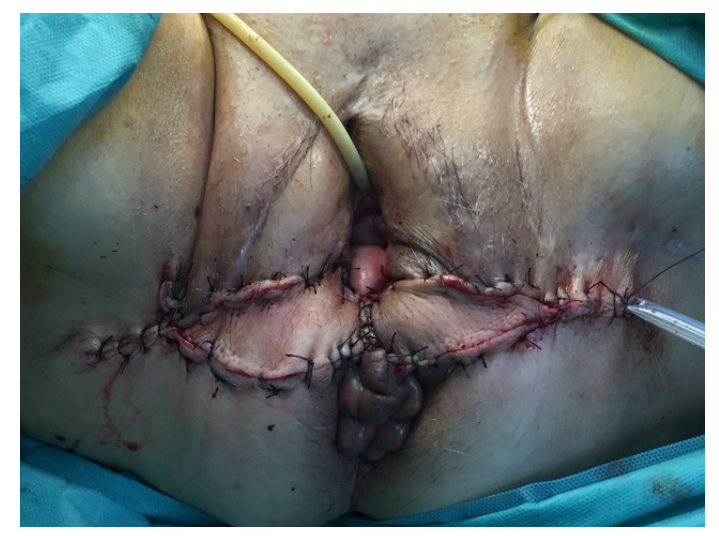

Figure 3 The local final appearance of the perineum after the removal of the recurrence and plastic reconstruction using lotus petal flaps.

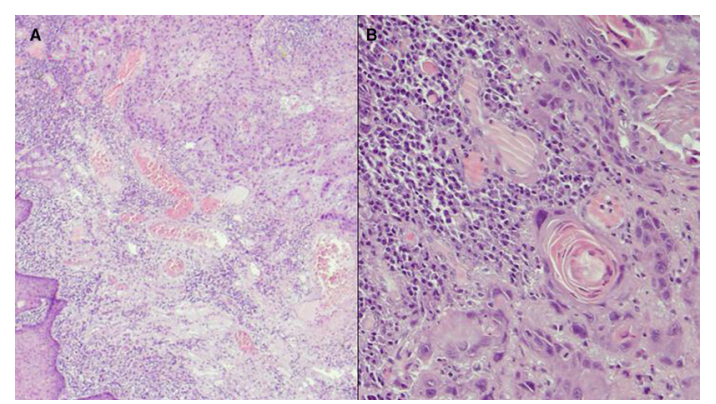

Figure 4 (H\&E, 10x A, 40 x B). Histological features of the tumor mass. solid sheets of cells grow with prominent accompanying vessels (A). The cells are rounded or spindled, with many atypical mitoses (B).

recurrence of an originally unknown component of the previous neoplasia. The original specimen was carefully reviewed, but the new cut sections did not show the presence of the undifferentiated component.

The occurrence of a mesenchymal neoplasia was taken into consideration, both because of the gross and microscopic morphology, and the expression of the immunohistochemical marker $\mathrm{CD} 31,{ }^{2}$ which is a very reliable feature of angiosarcoma. Moreover, epithelioid angiosarcomas can display a positive reaction to some antibodies against cytokeratins, among which are pancytokeratin and CK7. ${ }^{3}$ The lack of squamous morphology and of expression of associated transcription factors (p40, p63) was another element that contradicted the hypothesis of a recurrence. However, the gap of time between the removal of the primary tumor and the recurrence was very short in considering the development of an entirely new and different tumor, and the patient did not undergo radiation treatment that could have constituted a risk factor for sarcomas. Therefore, the ambiguous histology and immunological profile was interpreted favoring an undifferentiated component of carcinoma with sarcomatoid features and an aberrant expression of the vascular marker CD31.

Reactivity for CD31 in non-vascular tumors is rare and poorly addressed in the literature: it is thus considered a sensitive and specific marker of endothelial differentiation. Among the explanations proposed for this phenomenon is an immunohistochemical cross-reactivity with other membrane antigens, such as CEA, ${ }^{4}$ based on putative similarities between the two molecules, however, there is little evidence either to confirm or deny this hypothesis. Another theory is that aberrant expression of CD31 on non-endothelial

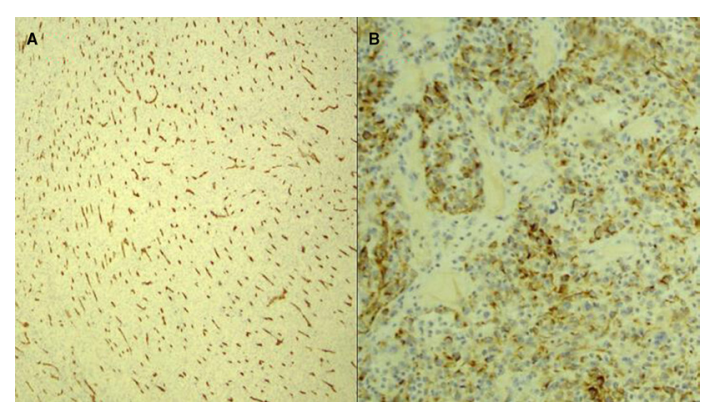

Figure 5 (IHC, $10 \times A, 20 \times B)$. In A, CD34 shows no reactivity on the tumor cells but underlines the vascular support structure. In B, the tumor shows membrane/ cytoplasmic positivity for cytokeratins. 


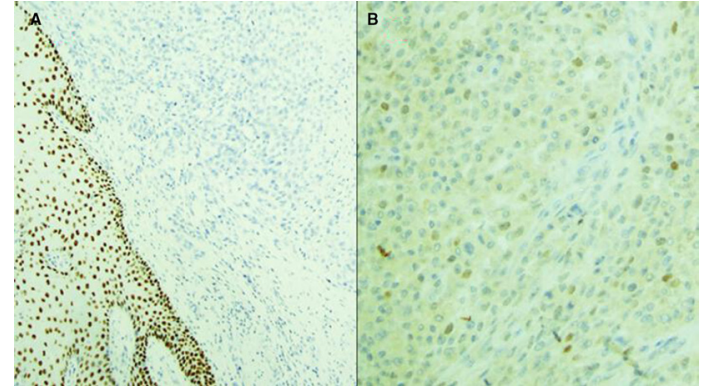

Figure 6 (IHC, 4x A, 10x B). Immunohistochemical expression of squamous differentiation, with no reactivity for p40 (internal positive control on normal squamous epithelium (A) and weak/focal positivity for p63 (B).

tumor cells is part of the series of modifications constituting the 'epithelial-mesenchymal transition', as it is reported in breast cancer, liver cancer, and non-Hodgkin's lymphomas to promote tumor cell invasion and metastases. ${ }^{5}$

\section{DR PADERNO AND DR ZAMBETTI}

Based on the pathological findings at the time of recurrence, what is the standard treatment recommendation?

From the available literature, 30 cases of sarcomatoid squamous cell carcinoma involving the vulva are reported. Median age at presentation was 77 years, and $60 \%$ of lesions involved the anterior vulva and clitoris. Tumors presenting with a more advanced stage have worse prognosis, demonstrating a rapid recurrence and progression even at an early stage. Noteworthy, seven patients have experienced a very rapid development of large local recurrences, followed by death and only six of 30 women were alive with a follow-up period of 24 to 60 months (four were stage I, one stage II, and one stage III disease). ${ }^{6-10}$

Considering the rarity of these tumors, no guidelines are available for vulvar squamous cell carcinoma with sarcomatoid features. In almost all cases, the primary treatment is surgical excision of the lesion, or radical vulvectomy with groin lymphadenectomy.

With the few cases in the literature, it is unclear which specific prognostic factors might dictate the need for additional therapy and if adjuvant chemotherapy or radiation therapy provides any benefit. However, the high risk of recurrence and the poor prognosis of this

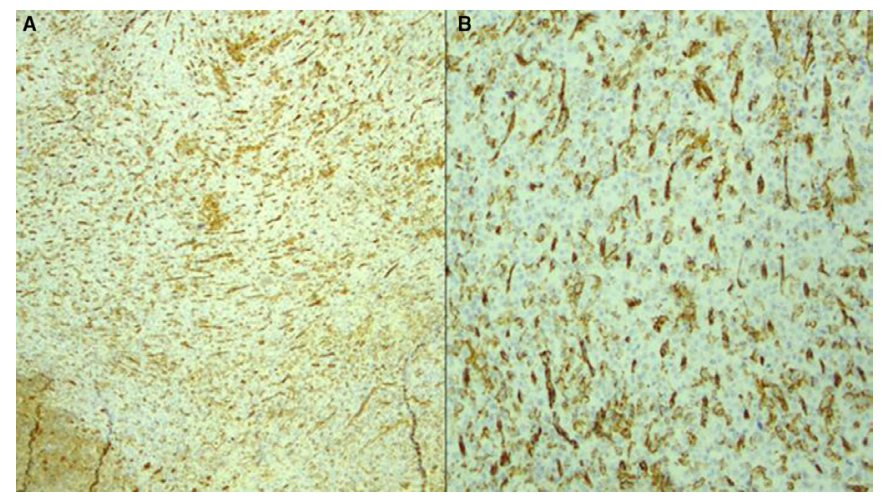

Figure 7 (IHC, 4x A, 10x B). CD31 marks normal vessels (A) but also shows strong membrane staining on tumor cells (B). type of tumor may support an aggressive strategy. If the patient's performance status and comorbidities allow, radiation therapy with or without chemotherapy may be performed as adjuvant therapy after primary surgery. In presence of distant metastasis chemotherapy is recommended.

Among the 30 cases reported thus far in the literature, seven patients received adjuvant treatment including radiotherapy with or without chemotherapy commonly including anthracyclines, taxanes, and ifosfamide. Notwithstanding, the rate of recurrence was the same as that for patients that did not receive further treatment. All patients died within 2 years. ${ }^{6-10}$

\section{DR COMERIO AND DR ZAMBETTI What would you consider the ideal recommendation after surgery for this patient?}

In our case the poor differentiation and the higher grade of aggressiveness of the neoplastic cells is an indicator of poor prognosis. Although, considering the age and co-morbidity of the patient presented here and the absence of distant metastasis, we proposed only clinical surveillance, and follow-up every 3 months was scheduled including physical examination and yearly CT scan. A more aggressive strategy including radiotherapy with concomitant chemotherapy would be preferred in a younger patient with a good performance status also to reduce the risk of recurrence.

\section{DR BUDA AND DR JACONI \\ Closing remarks}

Sarcomatoid squamous cell carcinoma is an uncommon variant of squamous cell carcinoma. This particular histotype is rare throughout all sites in the body, but the most commonly involved organs are the lung and the head and neck, where aggressive squamous cell carcinomas arise most frequently. In the female genital tract, it usually occurs in postmenopausal women and mostly in the cervix, rarely in the vulva or vagina, accounting for $1 \%-2 \%$ of all gynecologic malignancies. Risk factors for vulvar sarcomatoid squamous cell carcinoma are presumed to be the same as risk factors for conventional squamous cell carcinoma and include HPV infection, high-risk sexual behavior, cigarette smoking, prior radiotherapy, immunosuppression, history of precancerous vulvar conditions, or lichen sclerosus. ${ }^{9}$ As a result of the rarity of the disease and incomplete staging of many reported cases interpretation of available data is challenging. This subtype of vulvar cancer has an aggressive behavior with a propensity for both local recurrence and distant metastasis.

Regarding the gynecological tract, sarcomatoid carcinoma has to be distinguished from malignant mixed mullerian tumor, which arises in the corpus uteri. The fundamental difference between a sarcomatoid carcinoma and a mixed tumor (carcinosarcoma) is that the former is constituted by a single cell population of carcinomatous differentiation, although with the already discussed similarities with mesenchymal elements, and the latter is a biphasic tumor composed both of a carcinomatous component and a sarcomatous component which are morphologically and immunohistochemically distinct. Accurate clinico-pathological evaluation of both the primary and recurrent lesions is critical to differentiate between 


\section{Case study}

the possible ambiguous histology based on their immunological specific profile.

As previously discussed, such a brief lapse of time between removal of the original neoplasm and the occurrence of the second favors a regrowth of an aggressive, pre-existent component. It is also important to sample an adequate amount of any tumor mass of consistent size, because large neoplasms have more probability of harboring different and possibly dangerous subclones of cells that, even if present only in a focal area of the tumor, can adversely affect the prognosis.

Contributors All contributed to the conception and writing of the manuscript.

Funding The authors have not declared a specific grant for this research from any funding agency in the public, commercial, or not-for-profit sectors.

Competing interests None declared.

Patient consent for publication Not required.

Provenance and peer review Commissioned; externally peer reviewed.

\section{REFERENCES}

1 Confalonieri PL, Gilardi R, Rovati LC, et al. Comparison of V-Y advancement flap versus lotus petal flap for plastic reconstruction after surgery in case of vulvar malignancies: a retrospective single center experience. Ann Plast Surg 2017;79:186-91.
2 Lin F, Liu H. Immunohistochemistry in undifferentiated neoplasm/tumor of uncertain origin. Arch Pathol Lab Med 2014;138:1583-610.

3 Miettinen M, Fetsch JF. Distribution of keratins in normal endothelial cells and a spectrum of vascular tumors: implications in tumor diagnosis. Hum Pathol 2000;31:1062-7.

4 Simmons DL, Walker C, Power C, et al. Molecular cloning of CD31, a putative intercellular adhesion molecule closely related to carcinoembryonic antigen. J Exp Med 1990;171:2147-52.

5 Zhang Y-Y, Kong L-Q, Zhu X-D, et al. CD31 regulates metastasis by inducing epithelial-mesenchymal transition in hepatocellular carcinoma via the ITGB1-FAK-Akt signaling pathway. Cancer Lett 2018;429:29-40.

6 Bigby SM, Eva LJ, Jones RW. Spindle cell carcinoma of the vulva: a series of 4 cases and review of the literature. Int J Gynecol Pathol 2014;33:203-12.

7 Fiset PO, Wou K, Arseneau J, et al. Vulvar carcinosarcoma secondary to radiotherapy: a case report and review of the literature. $J$ Low Genit Tract Dis 2014;18:e74-9.

8 Koay MHE, Stewart CJR, Metastasis EM. Pericytic mimicry in sarcomatoid squamous cell carcinoma of the vulva: a report of 2 cases. Int J Gynecol Pathol 2019;38:27-31.

9 Choi D-S, Lee J-W, Lee S-J, et al. Squamous cell carcinoma with sarcomatoid features of the vulva: a case report and review of literature. Gynecol Oncol 2006;103:363-7.

10 Lordello L, Webb P, Oliva E. Vulvar carcinosarcoma composed of intestinal-type mucinous adenocarcinoma associated with anaplastic pleomorphic and spindle cell carcinoma and heterologous chondrosarcomatous and osteosarcomatous elements: a case report and review of the literature. Int J Gynecol Pathol 2018;37:93-100. 